\title{
On three parameters of invisibility graphs *
}

\author{
Josef Cibulka ${ }^{\dagger 1}$, Miroslav Korbelár̆ ${ }^{\ddagger 2}$, Jan Kynčl ${ }^{\S 1}$, Viola Mészáros ${ }^{\llbracket 3}$, Rudolf Stolar̆ ${ }^{11}$, and Pavel Valtr**1 \\ ${ }^{1}$ Department of Applied Mathematics and Institute for Theoretical Computer Science, Charles University, \\ Faculty of Mathematics and Physics, Malostranské nám. 25, 11800 Prague, Czech Republic \\ ${ }^{2}$ Department of Mathematics and Statistics, Faculty of Science, Masaryk University, Kotlářská 2, 61137 Brno, \\ Czech Republic \\ ${ }^{3}$ Institute for Mathematics, Technical University of Berlin, Strasse des 17. Juni 136, 10623 Berlin, Germany
}

\begin{abstract}
The invisibility graph $I(X)$ of a set $X \subseteq \mathbb{R}^{d}$ is a (possibly infinite) graph whose vertices are the points of $X$ and two vertices are connected by an edge if and only if the straight-line segment connecting the two corresponding points is not fully contained in $X$. We consider the following three parameters of a set $X$ : the clique number $\omega(I(X))$, the chromatic number $\chi(I(X))$ and the minimum number $\gamma(X)$ of convex subsets of $X$ that cover $X$.

We settle a conjecture of Matoušek and Valtr claiming that for every planar set $X, \gamma(X)$ can be bounded in terms of $\chi(I(X))$. As a part of the proof we show that a disc with $n$ one-point holes near its boundary has $\chi(I(X)) \geq \log \log (n)$ but $\omega(I(X))=3$.

We also find sets $X$ in $\mathbb{R}^{5}$ with $\chi(I(X))=2$, but $\gamma(X)$ arbitrarily large.
\end{abstract}

\section{Introduction}

Let $X$ be a subset of a $d$-dimensional Euclidean space. We say that two points $x, y \in X$ see each other if the straight-line segment $\overline{x y}$ connecting $x$ and $y$ is a subset of $X$. The invisibility graph $I(X)$ of a set

\footnotetext{
${ }^{*}$ This research was supported by the project CE-ITI (GACR P202/12/G061) of the Czech Science Foundation and by the grant SVV-2013-267313 (Discrete Models and Algorithms). The second author was supported by the project CZ.1.07/2.3.00/20.0003 of the Operational Programme Education for Competitiveness of the Ministry of Education, Youth and Sports of the Czech Republic. The fourth author was also partially supported by ESF EuroGiga project ComPoSe (IP03), by OTKA Grant K76099 and by OTKA Grant 102029. The first, the third and the sixth author were partially supported by project GAUK 52410. Part of the research was conducted during the Special Semester on Discrete and Computational Geometry at École Polytechnique Féderale de Lausanne, organized and supported by the CIB (Centre Interfacultaire Bernoulli) and the SNSF (Swiss National Science Foundation).

${ }^{\dagger}$ Email: cibulka@kam.mff.cuni.cz.

$\ddagger$ Email: miroslav.korbelar@gmail.com.

§Email: kyncl@kam.mff.cuni.cz.

ๆEmail: meszaros@math.tu-berlin.de.

" Email: ruda@kam.mff.cuni.cz.

**Email: valtr@kam.mff.cuni.cz.
}

$X \subseteq \mathbb{R}^{d}$ is a graph whose vertices are the points of $X$ and two vertices are connected by an edge if and only if they do not see each other. Let $\chi(G)$ be the chromatic number of a graph $G$ and let $\omega(G)$ be its clique number. For a set $X \subseteq \mathbb{R}^{d}$ we define $\gamma(X)$ to be the minimum possible number of convex subsets of $X$ that cover $X$. Further, let $\chi(X):=\chi(I(X))$ and $\omega(X):=\omega(I(X))$.

Sets $X$ with $\omega(X)=n-1$ are sometimes called $n$-convex [8].

Observe that $\omega(X) \leq \chi(X) \leq \gamma(X)$ for any set $X$.

If a planar set $X$ is closed, then $\gamma(X)$ can be bounded by a function of $\omega(X)$. This was proved by Breen and Kay [2] and the current best known upper bound is $\gamma(X) \leq O\left(\omega(X)^{3}\right)$ by Matoušek and Valtr [6]. From the other direction, there exist examples by Matoušek and Valtr [6] with $\gamma(X) \geq$ $\Omega\left(\omega(X)^{2}\right)$.

However, if we don't restrict ourselves to closed sets, there is no upper bound on $\gamma(X)$ even for sets with $\omega(X)=3$. An example is the disc $D_{\lambda}$ with $\lambda$ one-point holes punctured in the vertices of a regular convex $\lambda$-gon near the boundary of $D_{\lambda}$, for which $\omega\left(D_{\lambda}\right)=3$, but $\gamma\left(D_{\lambda}\right)=\lceil\lambda / 2\rceil+1$ (see $[6]$ ).

A one-point hole in a set $X \subset \mathbb{R}^{d}$ is a point that forms a path-connected component of $\mathbb{R}^{d} \backslash X$. Let $\lambda(X)$ be the number of one-point holes in the set $X$.

The example of the set $D_{\lambda}$ led to studying the properties of planar sets with a limited number of onepoint holes by Matoušek and Valtr [6]. In particular, they proved the following theorem.

Theorem 1 (Matoušek and Valtr [6]) Let $X \subseteq$ $\mathbb{R}^{2}$ be a set with $\omega(X)=\omega<\infty$ and $\lambda(X)=\lambda<\infty$. Then

$$
\gamma(X) \leq O\left(\omega^{4}+\lambda \omega^{2}\right)
$$

For any $\omega \geq 3$ and $\lambda \geq 0$ they also found sets $X$ with $\omega(X)=\omega, \lambda(X)=\lambda$ and $\gamma(X) \geq \Omega\left(\omega^{3}+\omega \lambda\right)$.

Matoušek and Valtr [6] conjectured that for an arbitrary planar set $X$, the value of $\gamma(X)$ is bounded by a function of $\chi(X)$. Then $\chi(X)$ cannot be bounded 
by a function of $\omega(X)$ as shows the above example with $D_{\lambda}$.

Lawrence and Morris [4] proved that for every $k$ there exists $n_{0}(k)$ such that whenever $S$ is a set of finitely many points in the plane and $|S| \geq n_{0}(k)$, then $\chi\left(\mathbb{R}^{2} \backslash S\right) \geq k .{ }^{1}$ Thus, whenever $X$ is the complement of a finite set of points, $\lambda(X)$ can be bounded in terms of $\chi(X)$. This implies, by Theorem 1, that the value of $\gamma(X)$ can be bounded in terms of $\chi(X)$, settling the conjecture of Matoušek and Valtr in the special case when $X$ is a complement of a finite set of points.

In this paper, we strengthen the result of Lawrence and Morris [4] and settle the conjecture for every planar set $X$.

The tower function $T_{l}(k)$ is defined recursively as $T_{0}(k)=k$ and $T_{h}(k)=2^{T_{h-1}(k)}$. Its inverse is the iterated logarithm $\log ^{(l)}(n)$, that is, $\log ^{(0)}(n)=n$ and $\log ^{(l)}(n)=\log \left(\log ^{(l-1)}(n)\right)$.

Theorem 2 Any set $X \subseteq \mathbb{R}^{2}$ with $\chi(X)=\chi<\infty$ satisfies

$$
\gamma(X) \leq O\left(2^{4 T_{2}(\chi)} \cdot \chi^{3}\right) .
$$

The proof of Theorem 2 is omitted from this extended abstract. In the full version of the paper, we also show that for every dimension $d, \lambda(X)$ can be bounded in terms of $\chi(X)$ for sets $X \subset \mathbb{R}^{d}$. This answers Question 6 of Lawrence and Morris [4].

A set $X$ is star-shaped if $X$ contains a point $x \in X$ that sees every other point of $X$.

In Sections 2 and 3 we show that $\chi$ and $\gamma$ can be separated in dimensions 5 and more.

Theorem 3 For every positive integer $g$ there exist star-shaped sets

1. $X \subset \mathbb{R}^{6}$ satisfying $\chi(X)=2$ and $\gamma(X) \geq g$ and

2. $X_{c} \subset \mathbb{R}^{6}$ that is closed and satisfies $\chi\left(X_{c}\right)=4$ and $\gamma\left(X_{c}\right) \geq g$.

Theorem 4 For every positive integer $g$ there exist star-shaped sets

1. $X \subset \mathbb{R}^{5}$ satisfying $\chi(X)=2$ and $\gamma(X) \geq g$ and

2. $X_{c} \subset \mathbb{R}^{5}$ that is closed and satisfies $\chi\left(X_{c}\right)=6$ and $\gamma\left(X_{c}\right) \geq g$.

Problem 1 Does there exist a function $f$ such that

$$
\gamma(X) \leq f(\chi(X)),
$$

1. for every set $X \subseteq \mathbb{R}^{3}$ ?

2. for every set $X \subseteq \mathbb{R}^{4}$ ?

All logarithms in this paper are binary. We use the notation $\overline{x y}$ for the straight line segment between points $x$ and $y$.

\footnotetext{
${ }^{1}$ The graph $\mathcal{G}_{S}$ in the paper of Lawrence and Morris is precisely the invisibility graph of $\mathbb{R}^{2} \backslash S$.
}

\section{Constructions in dimension 6}

\subsection{Set with chromatic number 2}

We prove part 1 of Theorem 3. Part 2 is omitted from this extended abstract.

Let $P_{n}$ be the cyclic polytope on $n \geq 7$ vertices (see for example [5]) and $V_{n}$ its set of vertices. Thus the convex hull of every triple of points from $V_{n}$ is a triangular face of $P_{n}$.

Lemma 5 Let $K_{n}$ be the complete graph on the set $V$ of $n \geq 7$ vertices and let $k:=\lceil 2 \log (n)+2\rceil$. It is possible to orient the edges of $K_{n}$ so that every set $V^{\prime} \subseteq V$ of size at least $k$ contains a directed triangle.

Proof. For brevity, we call a set $V^{\prime}$ good if it contains a directed triangle.

We orient the edges randomly and show that with positive probability, every set $V^{\prime} \subseteq V$ of size at least $k$ is good.

First, we will bound the probability $b_{k}$ that a given set $V^{\prime}$ of $k$ vertices is bad.

If there exists a directed cycle on $V^{\prime}$ of length greater than 3 , then one of the two cycles created by adding an arbitrary diagonal to the cycle is again directed. Thus there exists a directed triangle on $V^{\prime}$.

There are $2^{k(k-1) / 2}$ possible orientations of edges of a complete graph on $k$ vertices out of which $k$ ! are acyclic. Thus

$$
b_{k}=\frac{k !}{2^{k(k-1) / 2}}=k ! 2^{-k^{2} / 2+k / 2} .
$$

The probability that some $k$-tuple $V^{\prime}$ of vertices is bad is thus at most

$$
\begin{aligned}
\left(\begin{array}{l}
n \\
k
\end{array}\right) b_{k} & \leq \frac{n^{k}}{k !} b_{k}=2^{k \log (n)-k^{2} / 2+k / 2}= \\
& =2^{k(\log (n)-k / 2+1 / 2)} \leq \\
& \leq 2^{k(\log (n)-\log (n)-1+1 / 2)} \leq 2^{k(-1 / 2)}<1 .
\end{aligned}
$$

We fix the orientation of the edges of $P_{n}$ in which every $k$-tuple of vertices is good. A triangular face of $P_{n}$ has directed boundary if the three edges of the face form a directed cycle. The set $X$ is constructed by puncturing a one-point hole in the barycenter of triangular faces of $P_{n}$ with directed boundary.

Vertices of $P_{n}$ are colored black. Edges are cut in thirds. In every edge, the interior of the middle third together with the point at one third closer to the end of the edge is colored white. The rest of the edges is black. The coloring of triangular faces with directed boundary is depicted on Fig. 1. The rest of $X$ is colored black.

All the edges of the invisibility graph of $X$ are between pairs of points lying on the same triangular face 


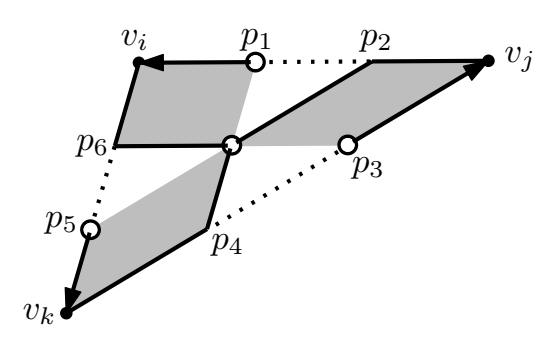

Figure 1: Coloring of a face with directed boundary. Lines determined by pairs $\left(p_{1}, p_{4}\right),\left(p_{2}, p_{5}\right)$ and $\left(p_{3}, p_{6}\right)$ intersect in the barycenter and split the triangle into monochromatic regions. Full lines and gray areas represent black color, the rest is white.

with directed boundary. The coloring is proper on each of these faces and thus the 2-coloring of the whole $X$ is proper.

If a convex set $C$ contains at least $k$ vertices of $X$, then it contains a triangular face with directed boundary and thus $C$ contains a one-point hole. Therefore

$$
\gamma(X) \geq \frac{n}{2 \log (n)+3}
$$

\section{Constructions in dimension 5}

Here we present some parts of the proof of part 1 of Theorem 4. The rest of the proof is omitted from this extended abstract.

The constructions are similar to those in dimension 6: for part (1) of the theorem, the set $X$ is a closed cyclic polytope with one-point holes in some of the 2-dimensional faces. For part (2), instead of points we remove small 5-dimensional polytopes attached to the 2-dimensional faces. The difference from the construction in dimension 6 is in the placement of the holes: here we cannot apply the same argument as in the previous section since for the cyclic polytope in dimension 5 only quadratically many triples of vertices induce a 2-dimensional face and there is a 2-coloring of the vertex set in which no 2-dimensional face is monochromatic.

In the full version of the paper, we show two different ways how to choose the holes. In the first construction we essentially show that randomly chosen holes will do, but the proof (interestingly) requires a rather nontrivial result from group theory. Also the construction proves only part (1) of the theorem. In the second construction we specify the locations of the holes precisely. Moreover, we show that the holes can be enlarged to open pyramids, which shows part (2) of the theorem. The proof of part (2) of the theorem is analogous to the proof of part (2) of Theorem 3 and is omitted from this abstract.

Let $P_{n}$ be the 5 -dimensional cyclic polytope on $n \geq 6$ vertices with (ordered) vertex set $V_{n}=$ $\left\{v_{1}, v_{2}, \ldots, v_{n}\right\}$. For brevity, we call the triangular face with vertices $v_{i}, v_{j}$ and $v_{k}$ the $i j k$ triangle. Similarly, the $i j$ edge is the edge between vertices $v_{i}$ and $v_{j}$. The 2-dimensional faces of $P_{n}$ are the triangles

- $1 i j$ for every $1<i<j \leq n$ (type $1 i j$ triangles),

- $i j n$ for every $1 \leq i<j<n$ (type $i j n$ triangles),

- $i(i+1) j$ for every $1<i<j-1<n$ (type $i(i+1) j$ triangles) and

- $i j(j+1)$ for every $1<i<j<n-1$ (type $i j(j+1)$ triangles).

\subsection{Covering with convex sets}

In the constructions proving part (1) of Theorem 4, we remove a one-point hole from every type $1 i j$ triangle. In the construction proving part (2), we remove an open flat simplex instead of the point (as in Section 2). The following lemma shows that in both cases, the resulting set can not be covered by a bounded number of convex sets.

Lemma 6 Let $X$ be a subset of $P_{n}$ such that every edge of $P_{n}$ is a subset of $X$ and none of the type 1 ij triangles is a subset of $X$. Then $\gamma(X) \geq$ $\Omega(\log n / \log \log n)$.

Proof. Let $X=C_{1} \cup C_{2} \cup \cdots \cup C_{k}$ be a covering of $X$ with convex subsets of $X$. The covering induces a partition of each open edge $1 i, 2 \leq i \leq n$, into at most $k$ intervals $I_{i}^{1}, I_{i}^{2}, \ldots, I_{i}^{k_{i}}$, where each of the intervals $I_{i}^{j}$ is covered by one of the convex sets $C_{l(i, j)}$. Since the convex sets in the covering may overlap, this partition need not be unique; in such a case we just pick one.

We say that the partitions of two edges $1 i$ and $1 i^{\prime}$ are of the same type if $k_{i}=k_{i^{\prime}}, l(i, p)=l\left(i^{\prime}, p\right)$ for each $p=1,2, \ldots, k_{i}$ (in other words, the "colors" appear in the same order along the edges), and for each $p=1,2, \ldots, k_{i}$ the type of the interval $I_{i}^{p}$ (that is, closed, open, or half-closed from the left/right) is the same as the type of the interval $I_{i^{\prime}}^{p}$. Degenerate onepoint intervals are considered as closed. The number of types of the partitions is at most $2^{k} \cdot k ! \cdot 2^{k-1}$. Indeed, there are at most $2^{k}$ subsets of "colors", each of the subsets can be linearly ordered in at most $k$ ! ways, and there are at most $k-1$ boundary points shared by two intervals, where one of the intervals is locally closed and the other one locally open.

It follows that if $n>2^{k} \cdot k ! \cdot 2^{k-1}+1$, then there are two edges $1 i$ and $1 i^{\prime}$ of the same type. The convex hulls $\operatorname{conv}\left(I_{i}^{p} \cup I_{i^{\prime}}^{p}\right)$ cover the whole open triangle $1 i i^{\prime}$, including the one-point hole inside, which is a contradiction. Therefore $n \leq 2^{k} \cdot k ! \cdot 2^{k-1}+1$, which implies that $\gamma(X) \geq \Omega(\log n / \log \log n)$. 


\section{Concluding remarks}

To solve Problem 1 in dimension 4 we could use a construction similar to those in dimensions 5 and 6 provided the following problem has a positive answer.

Problem 2 Does there exist for every $k$ a convex simplicial polytope $P(k)$ in $\mathbb{R}^{4}$ such that in every coloring of vertices of $P(k)$ by $k$ colors we can find a triangular face whose vertices are monochromatic?

Assuming the polytope $P(k)$ from Problem 2 exists, the set $X$ from Problem 1 is obtained from $P(k)$ by making a one-point hole in an arbitrary point inside every triangular face. Such a set $X$ cannot be covered by $k$ convex sets since otherwise one of the convex sets would contain three vertices of a triangular face.

The invisibility graph $I(X)$ can be colored by 13 colors in the following way. All the vertices of $P(k)$ get color 1. Tancer [7] has shown that the edges of every 2-dimensional simplicial complex PL-embeddable in $\mathbb{R}^{3}$ can be colored by 12 colors so that for every triangular face, the three edges on its boundary have three different colors. This applies, in particular, to the 2-skeleton of every 4-dimensional convex simplicial polytope. We use colors $2,3, \ldots, 13$ to color the interiors of edges of $P(k)$ in this way. For each triangular face and each point $p$ on its boundary, the interior of the segment conecting the one-point hole with $p$ is colored by the color of $p$. All the remaining points of $X$ are isolated in $I(X)$ and thus may be colored arbitrarily.

The boundary complex of a 4-dimensional convex simplicial polytope is a special case of a triangulation of $S^{3}$. If we relax the condition on polytopality in Problem 2 and ask only for a triangulation of $S^{3}$, then the answer is yes. Heise et al. [3] constructed, for every $k$, a 2-dimensional simplicial complex linearly embedded in $\mathbb{R}^{3}$ such that in every coloring of its vertices with $k$ colors at least one of the triangles is monochromatic. We found the same simplicial complex independently, modifying Boris Bukh's construction, which was communicated to us by Martin Tancer. The vertices of the complex are placed on the moment curve and a suitable noncrossing subset of triangles is chosen for the faces. It remains to extend the embedded complex to a triangulation of the whole $\mathbb{R}^{3}$, or $S^{3}$ [1].

\section{References}

[1] K. Adiprasito, F. Lutz and J. Moller, unpublished manuscript.

[2] M. Breen and D. C. Kay, General decomposition theorems for $m$-convex sets in the plane, Israel Journal of Mathematics 24 (1976), 217-233.
[3] C. G. Heise, K. Panagiotou, O. Pikhurko and A. Taraz, Coloring $d$-embeddable $k$-uniform hypergraphs, arXiv:1209.4879 (2012).

[4] J. Lawrence and W. Morris, Finite sets as complements of finite unions of convex sets, Discrete 83 Computational Geometry 42 (2009), no. $2,206-218$.

[5] J. Matoušek, Lectures on Discrete Geometry, Springer-Verlag, New York (2002).

[6] J. Matoušek and P. Valtr, On visibility and covering by convex sets, Israel Journal of Mathematics 113 (1999), no. 3, 341-379.

[7] M. Tancer, unpublished manuscript.

[8] F. Valentine, A three point convexity property, Pacific Journal of Mathematics 7 (1957), no. 2, 1227-1235. 